Álvarez-Cooper, O.L y Rivera-González, J.G. (2018). Condiciones laborales y emancipación juvenil en el siglo XXI: reflexiones desde Latinoamérica. Revista de Antropología y Sociolgía: VIRAJES, 20(2) 35-53. DOI: 10.17151/rasv.2018.20.2.3.

\title{
Condiciones laborales y emancipación juvenil en el siglo XXI: reflexiones desde Latinoamérica*
}

\author{
OLGA LETICIA ÁLVAREZ-COOPER** \\ JOSÉ GUADALUPE RIVERA-GONZÁLEZ***
}

Recibido: 20 de febrero de 2018

Aprobado: 20 de marzo de 2018

Artículo de Reflexión

\footnotetext{
* Este artículo hace parte del proyecto en curso denominado "Teatro y emancipación juvenil: el caso de San Luis Potosí, México y Manizales, Colombia", desarrollado dentro del marco del Programa de Estudios Latinoamericanos en Territorio, Sociedad y Cultura de la UASLP, México. Posgrado perteneciente al PNPC de CONACYT.

** Candidata a Doctora en Estudios Latinoamericanos en Territorio, Sociedad y Cultura, Facultad de Ciencias Sociales y Humanidades de la Universidad Autónoma de San Luis Potosí. Maestra en Educación, Universidad del Centro de México. Licenciada en Comercio Exterior, Facultad de Economía de la Universidad Autónoma de San Luis Potosí. E-mail: 1lqper@alumnos.uaslp.edu.mx. (1) ORCID: 0000-0003-1909-7431. Google Scholar *** Antropólogo, Maestro y Doctor en Ciencias Antropológicas, Departamento de Antropología de la Universidad Autónoma Metropolitana, Unidad Iztapalapa. Profesor-investigador de tiempo completo en la Facultad de Ciencias Sociales y Humanidades de la Universidad Autónoma de San Luis Potosí, México. E-mail: joserivera@uaslp.mx. @ ORCID: 0000-0002-2108-3963. Google Scholar
} 


\title{
Resumen
}

Este artículo forma parte de la revisión bibliográfica de una investigación más amplia; nuestro objetivo es comprender cómo los modelos laborales del siglo XXI han transformado las trayectorias de emancipación familiar y económica que se suponía inauguraban el tránsito de la condición juvenil a la adulta. Cuestionamos las categorías de joven y adulto ligadas al mundo laboral para entender cómo han ido cambiando en el contexto latinoamericano. La revisión documental realizada nos permite reflexionar sobre uno de los aspectos señalados con mayor frecuencia en los análisis sobre los jóvenes: la insuficiencia de oportunidades laborales dignas, situación que dificulta su transición a la vida adulta. La independencia económica se ha vuelto cada vez más difícil de conseguir; la precariedad laboral ha ralentizado la llegada a la adultez. Ante este panorama, muchos jóvenes han desarrollado nuevas estrategias para resolver la tensión de acceder a espacios laborales y crear condiciones para su emancipación.

Palabras clave: jóvenes, emancipación, empleo, condiciones laborales.

\section{Working conditions and youth emancipation in the 21st century: reflections from Latin America}

\begin{abstract}
This article is part of the bibliographical review of a broader research. The main objective is to understand how the labor models of the 21st century have transformed the trajectories of family and economic emancipation that were supposed to inaugurate the transition from youth to adulthood. The categories of young people and adults are questioned linked to the world of work to understand how they have changed in the Latin American context. The documentary review carried out allowed the reflection on one of the aspects most frequently mentioned when analyzing young people: the lack of decent work opportunities, a situation that hinders their transition to adult life. Economic independence has become increasingly difficult to achieve; labor precariousness has slowed down the arrival to adulthood. Given this panorama, many young people have developed new strategies to resolve the tension of accessing work spaces and creating conditions for their emancipation.
\end{abstract}

Key words: youth, emancipation, employment, working conditions. 


\section{Introducción}

E 1 presente artículo es producto de la revisión de trabajos generados en años recientes en donde el tema central es la reflexión sobre la situación laboral de los jóvenes en América Latina. Lo que se deriva de esas investigaciones es que actualmente los jóvenes son un grupo especialmente vulnerable en lo que se refiere a las condiciones de empleo; experimentan alta rotación y los montos que perciben por sus salarios son inferiores en comparación con la población adulta. En la mayoría de los países, los jóvenes inician su vida laboral teniendo muchas probabilidades de enfrentarse al desempleo por amplios periodos, percibir bajos salarios y carecer de prestaciones. Entender las transformaciones suscitadas en el mundo laboral resulta clave para entender las complejidades de este tema que no sólo ha impactado a los jóvenes en las llamadas economías emergentes, sino que también es ya un grave problema de las economías desarrolladas, resultado de los efectos de la crisis del 2008.

El texto se encuentra organizado en los siguientes apartados: el primero, La edad desde la perspectiva antropológica. Aquí la parte central de la revisión bibliográfica llevará al lector a conocer la manera en la que se abordó y construyó el concepto de juventud desde las ciencias sociales, pasando por las aproximaciones centradas en la perspectiva biopsicologista hasta llegar a una construcción en donde el concepto de juventud pasa por la mirada de lo cultural. El segundo apartado lo hemos titulado Jóvenes $e$ inserción laboral. La parte central de este segundo apartado radica en documentar, a través de datos recabados en diversas publicaciones, que la población joven en el mundo, en particular en América Latina, enfrenta un panorama poco favorable para llevar a cabo su inserción al mercado laboral. El tercer y último apartado lleva por título Flexibilidad laboral y juventudes latinoamericanas, aquí destacamos que uno de los aspectos señalados con mayor frecuencia en los análisis sobre los jóvenes latinoamericanos es la insuficiencia de oportunidades laborales dignas; por lo tanto, esta situación dificulta su inclusión y cohesión social, y, por ende, su transición a la vida adulta. Así es como reflexionamos sobre las categorías de adulto y joven ligadas al mundo laboral para comprender cómo han ido cambiando en el contexto latinoamericano y cuáles han sido las estrategias desarrolladas desde el mundo de los jóvenes para resolver la tensión de acceder o crear espacios laborales. 


\section{Trabajo y emancipación juvenil}

\section{La edad desde la perspectiva antropológica}

La edad ha servido históricamente como uno de los principios organizadores de la vida en sociedad (Feixa, 1999). La aproximación antropológica al estudio de la edad derivó en concebírsele como una construcción cultural dependiente de diferentes factores como: estructura, espacio y temporalidad propios de cada grupo social. Al analizar las trayectorias vitales de las personas, se pueden identificar etapas fisiológicas y culturales que demarcan el desarrollo de los individuos. Los periodos caracterizados por "contenidos culturales" (Feixa, 1999, p. 2) particulares son precisamente objeto de estudio antropológico. A través de esta disciplina se pretende conocer los elementos culturales establecidos por cada estructura social con la intención de instaurar conductas y organizar a las personas en un espacio y tiempo específicos. El estudio antropológico de sujetos y grupos localizados en diversas coordenadas del mundo confirmó el "carácter relativo de la división de las edades" (Feixa, 1999, p. 2), lo anterior llevó a entender que edad biológica y edad social se desarrollan a su propio ritmo y que no siempre concurren.

La perspectiva de la edad vista como una construcción cultural ha originado un cuerpo de conocimientos que pudiera nombrarse como "Antropología del ciclo vital y de las relaciones intergeneracionales" (Feixa, 1999, p. 3). Esta vertiente disciplinar ha centrado su mirada con creciente interés en los jóvenes. El estudio de la juventud se remonta al siglo XIX; las primeras aproximaciones a los sujetos jóvenes fueron bajo las gafas de la medicina, la biología y la psicología. Los constructos teóricos del desarrollo humano y la socialización fueron los ejes que permitieron inaugurar los conocimientos científicos sobre este grupo de edad. A partir de dichas posturas se llegó a la conclusión de que la adolescencia era una "etapa universal del desarrollo biológico y mental" (Urteaga, 2009, p. 14), por la que transitaba cualquier individuo antes de llegar a la edad adulta. Dicha etapa suponía la inmadurez como una de sus características; concepción que justificó la contención de los jóvenes en instituciones administradas y vigiladas por los adultos. La escuela fue uno de esos lugares consignados para preparar y guiar a los sujetos jóvenes hacia el mundo adulto.

Juventud se entendía en ese entonces como una etapa del desarrollo de una persona, entendido ese desarrollo como proceso cronológico lineal cuyo culmen era convertirse en adulto. En esa época había dos miradas contrapuestas sobre la etapa juvenil; por un lado, se le veía como una 
amenaza, como una distorsión de lo que debiera ser un adulto. Así también había quienes eran más optimistas al concebir a los jóvenes como buenos pero vulnerables, incluso se les pensaba como la esperanza del futuro (Urteaga, 2009). Esta ambigüedad en la comprensión del sujeto joven legitimó la tutela del Estado, que fue entonces el encargado de controlar el camino que llevaba de la infancia a la edad adulta.

Algunos trabajos etnográficos llevados a cabo por investigadores de la Escuela de Chicago (Thrasher, 1963; Whyte, 1971) fueron clave para impulsar los estudios sobre jóvenes urbanos en el siglo XX. En un primer momento, estudiaron agrupaciones callejeras conformadas por jóvenes; bandas o pandillas que causaban preocupación y rechazo pues se les veía como sujetos inestables, desequilibrados y, por lo tanto, peligrosos. El conocimiento producido por la Escuela de Chicago tuvo gran relevancia en la producción de estudios culturales llevados a cabo por la Escuela de Birmingham a partir de la década de 1970; la cual se interesó, en particular, en las prácticas culturales, en la dimensión material y simbólica de las experiencias juveniles. Las diferentes conductas, gustos, costumbres, formas de vestir y de comunicarse observadas en las agrupaciones juveniles analizadas desencadenaron que desde el ámbito académico se comenzara a ver a los jóvenes también como creadores de cultura (Urteaga, 2009).

A pesar del interés por conocer las expresiones culturales de los jóvenes, la Escuela de Birmingham generó cuestionamientos en la comunidad científica por orientarse principalmente al estudio de "lo desviado" (Feixa, 1999) en hombres jóvenes que, en su mayoría, pertenecían a la clase obrera. Aunque sesgada, la visión sobre los jóvenes aportada por dicha escuela sirvió como detonador de nuevos estudios etnográficos enfocados a conocer las vidas cotidianas de los sujetos y rescatar las distintas voces de los actores con la intención de comprender a fondo el fenómeno mundial del surgimiento de "microculturas juveniles" (Feixa, 1999). Ya fuesen integradas, indiferentes o contrarias a sus respectivos sistemas, la "emergencia de estilos juveniles espectaculares" (Feixa, 1999) comenzó a interpretarse como una señal que advertía sobre transformaciones sociales en marcha.

En el caso particular de México, desde inicios de la segunda mitad del siglo XX, lo juvenil ganó interés investigativo; generaban inquietud aquellos jóvenes que habitaban zonas marginadas y que se unían para formar grupos callejeros o pandillas. Posteriormente, durante la década de 1960, investigaciones como Los hijos de Sánchez de Óscar Lewis (2012) se orientaron a otros grupos juveniles; esto permitió modificar de forma gradual la concepción que sobre ellos se tenía (Urteaga, 2009). Se les 
empezó a ver como sujetos con capacidad de agencia sociocultural. En ese sentido, el presente trabajo se adscribe a la perspectiva de análisis sugerida por Feixa (1999), la cual consiste en entender que la cultura es dinámica y cada generación va aportando a su construcción continua. A partir de esa premisa, se concibe entonces a los jóvenes como partícipes y creadores de procesos de producción, uso y difusión de contenidos culturales, y, en consecuencia, también puede mirárseles como sujetos implicados en cambios sociales.

\section{Jóvenes e inserción laboral en América Latina}

\section{El valor del empleo formal: La experiencia en la década de los 70 y 80 en América Latina}

En la mayoría de los países de América Latina en la década de los 60 y 70 del siglo XX las economías experimentaron importantes niveles de crecimiento; esto se tradujo en ciertas mejoras al bienestar de la clase trabajadora, la cual recibió los beneficios de ese importante crecimiento económico. Sin embargo, estas mismas economías experimentarían una crisis, la cual derivó en la llamada década perdida. Dicha crisis ocasionó reducción de puestos de trabajo en varias ramas de la actividad productiva, aumento de la informalidad y de empleos precarios, e incremento del desempleo; por lo tanto, todo lo anterior agudizó la desigualdad social y la pobreza.

Este contexto de reajuste de políticas económicas y adecuación de los mercados de trabajo sirvió como escenario de diversas investigaciones realizadas durante las últimas tres décadas en diferentes países del continente americano; las cuales documentaron el valor que representó contar con empleos en empresas formales, lo que contribuyó a la consolidación de rutinas diarias o roles que serían desempeñados por cada uno de los miembros de las familias. De esta manera, fue que se documentó el papel de hombres y mujeres como proveedores de recursos económicos. También se pudo constatar que tener un empleo estable posibilitó, a quien lo tuviera, la seguridad de contar con un ingreso/ salario seguro y permanente durante largos periodos de tiempo. Además, millones de trabajadores contaron con respaldo sindical, lo cual les brindaba posibilidades de contar con una representación encargada de defender sus intereses laborales. Trabajar también otorgaba sentido de bienestar; esto se traducía en gozar de buena salud emocional. Además, otorgaba un sentido del deber y de la responsabilidad. De igual manera, el trabajo posibilitó que se pudiera 
contar con una amplia red de convivencia y de apoyos dentro y fuera del espacio laboral. Es decir, el trabajo generaba ingreso económico y ese ingreso permitía que se tuviera acceso a una amplia red social y que se estuviera en posibilidades de acceder a un patrimonio familiar en forma de casa, autos, joyas, viajes y otros beneficios personales, pero sobre todo, beneficios materiales (Bazán,1996; Estrada, 1996; García Díaz, 1981; García y De Oliveira, 1994; González de la Rocha,1986; Gutmann, 1993; Nieto 1988; Novelo y Urteaga 1980; Oliveira y Salles, 1989; Safa, 1999; Rivera González, 2004).

\section{Las crisis económicas de los noventa y su impacto en las experiencias laborales de los jóvenes en América Latina}

Es un hecho que las condiciones económicas de la mayoría de los países latinoamericanos sufrieron cambios notables en la década de los ochenta y noventa y esos cambios, sin duda, repercutieron en el mercado de trabajo y, por ende, en las condiciones de acceso a un empleo entre las nuevas generaciones (Contreras, 2015; Escalante, 2015). Esta situación ha repercutido de forma negativa en las condiciones bajo las cuales las familias llevan a cabo su reproducción social. Como resultado del estancamiento en la actividad económica, durante los últimos años se ha observado un incremento notable del individualismo, un importante deterioro de las relaciones de solidaridad familiar $\mathrm{y}$, en algunos casos, la exclusión social; además de haberse redefinido las relaciones laborales, para hombres, mujeres, jóvenes y adultos mayores, quienes han visto incrementado las jornadas laborales en condiciones adversas, mientras que, en otros casos, se ha enfrentado a la carencia de un trabajo remunerado durante periodos considerables de tiempo (Castillo, Baca y Todaro, 2016; Macri, 1999; Mancini, 2017; Saraví, 2009; Trejo Sánchez, Arzate y Palermo, 2010).

Como bien lo documentaron varias de las investigaciones arriba señaladas, los jóvenes resultaron ser uno de los sectores testigos de cómo las oportunidades laborales estables y decentes se alejaban de sus posibilidades. Al respecto, conviene agregar que el año de 1985 fue declarado por la UNESCO como Año Internacional de la Juventud. Este nombramiento era una "señal de alarma" (Feixa, 2006, p. 12) de que las cosas no iban del todo bien para los jóvenes. Dicha declaración se daba en un momento en el que el paro laboral juvenil había incrementado, las corrientes ideológicas contrarias al sistema hegemónico habían perdido fuerza y, además, aparecía el fenómeno de los jóvenes con dificultades para emanciparse de sus familias. Ese diagnóstico trasladó los discursos que antes hablaban sobre "la capacidad revolucionaria y constructiva" (Feixa, 
2006) de los jóvenes, hacia el terreno de la preocupación por dos temas ligados estrechamente a la juventud: desempleo e incertidumbre.

Una vez que dio inicio el nuevo sigloen América Latina, las condiciones laborales para los jóvenes no auguraban mejoras en comparación con las décadas pasadas. En la primera década del siglo XXI la Comisión Económica para América Latina y el Caribe (CEPAL) en conjunto con la Organización Iberoamericana de la Juventud (OIJ) publicaron el documento: La juventud en Iberoamérica: Tendencias y urgencias (CEPAL, OIJ, 2007). Este fue un texto clave para entender muchas de las incertidumbres que enfrentaban en ese momento y que seguirían acarreando en el futuro los jóvenes latinoamericanos en diferentes ámbitos de su vida cotidiana; uno de estos ámbitos era el mundo laboral.

$\mathrm{Al}$ respecto, este documento destacó que los jóvenes tienen más acceso a la educación, pero contradictoriamente estos mismos jóvenes tienen menos oportunidades de empleo. Es decir, millones de jóvenes en América Latina tienen en la actualidad una mayor escolaridad en comparación con las anteriores generaciones, pero a la vez enfrentan y padecen altos porcentajes de desempleo. Estas nuevas generaciones han adquirido mayores conocimientos, pero a la vez se encuentran cada vez más excluidos del mundo laboral y de fuentes de trabajo que permitan generar ingresos económicos capaces de mejorar sus condiciones de vida. A diferencia de las anteriores generaciones, hoy contar con una mayor educación no se traduce en mayor estabilidad laboral, tampoco en movilidad social.

Dentro de la amplia gama de trabajos publicados sobre las problemáticas laborales de la población joven, el texto de Jürgen Weller (2007) destacó que son varios los puntos que han contribuido a que se vuelva cada vez más difícil y complejo acceder a un empleo para los jóvenes latinoamericanos. Este autor destacó que en ello han jugado un papel clave no sólo las transformaciones económicas que han planteado nuevos retos al mercado laboral. También los nuevos desarrollos tecnológicos han desempeñado un rol muy importante, contribuyendo a la flexibilidad del mercado de trabajo y de la mano de obra. En sociedades con marcadas desigualdades económicas estaremos siendo testigos de los problemas que encararán los jóvenes para lograr construir carreras laborales duraderas y exitosas.

Además de factores de índole económico y tecnológico, hay otras circunstancias que pueden dificultar la posibilidad de los jóvenes de acceder a un empleo: a) el trasfondo familiar determina en gran medida las perspectivas laborales de los jóvenes, pues influye en las oportunidades para acumular capital humano (acceso a educación y capacitación de buena calidad); b) el capital social (relaciones sociales basadas en la confianza, 
la cooperación y la reciprocidad); y, c) el capital cultural (manejo de los códigos establecidos por la cultura dominante) (Weller, 2007). Esto hace que situaciones, problemas y perspectivas relacionadas con el mundo del trabajo de los jóvenes se diversifiquen volviéndose sumamente heterogéneas.

En años más recientes, la Oficina Internacional del Trabajo (OIT, 2015) informó que el número de desempleados jóvenes en el mundo en 2014 se redujo en comparación con la cifra reportada cinco años atrás (de 76,6 millones a 73,3 millones). Lo anterior ratifica que "no es fácil ser joven en el mercado de trabajo actual" (OIT, 2015, p. 1). La frase anterior hace alusión a la persistencia de la crisis del empleo para la población joven en diferentes regiones del mundo. Por ejemplo, en países de Europa Central, Latinoamérica, el Caribe y África Subsahariana, las tasas de desempleo juvenil han demostrado tendencia decreciente tanto en el mediano como en el corto plazo, pero también es un hecho que más gente joven trabaja en forma parcial o temporal en combinación con la educación. Los datos presentados para 2015 mostraron que 2 de cada 5 jóvenes económicamente activos $(42,6 \%)$ seguían desempleados y los que trabajaban no lograban superar las condiciones de pobreza. A pesar de la disminución en la tasa mundial de desempleo juvenil, en demasiados países la población joven sigue sufriendo las consecuencias de la crisis económica y de las reformas políticas instauradas para hacerle frente. En estos países, para un joven sin experiencia laboral encontrar trabajo, y más aún trabajo a tiempo completo, es una lucha ardua y larga. Amplios porcentajes de la población joven se involucran en actividades laborales de tipo temporal (menos de 30 horas a la semana). El trabajo a tiempo parcial en los países de bajos ingresos puede interpretarse principalmente por la irregularidad del horario laboral de los trabajadores jóvenes más vulnerables (trabajadores por cuenta propia y trabajadores familiares auxiliares), así como de los trabajadores ocasionales remunerados.

En términos laborales, los datos de la OIT demuestran que los jóvenes con trabajo tenían 1,5 veces más probabilidades de encontrarse en situación de pobreza extrema que los adultos y 1, 2 más posibilidades de encontrarse en situación de pobreza moderada. Los adultos tenían más posibilidades de encontrarse en los grupos de clase media en desarrollo y de nivel superior. Los jóvenes enfrentan una situación de marcada vulnerabilidad en lo que se refiere a las condiciones laborales: trabajadores irregulares, ya sea como trabajadores por cuenta propia, trabajadores familiares auxiliares, trabajadores ocasionales remunerados o trabajadores temporales (no ocasionales). Un sector de jóvenes ha incrementado su nivel educativo, sin embargo, no toda la gente joven se beneficia de ello. Millones de jóvenes en países de bajos ingresos siguen abandonando la escuela prematuramente 
para trabajar desde edad temprana. No hay duda de que millones de jóvenes alrededor del mundo han tenido acceso a empleos, sin embargo, para una gran parte de esos jóvenes, los empleos a los que han accedido son empleos precarios y mal pagados que no les brindan posibilidades para transitar a un bienestar económico. Los países que no establezcan condiciones para que los mercados generen empleos estables y bien remunerados para los jóvenes, difícilmente crecerán, ya que los empleos actualmente ofertados no aseguran el desarrollo de los jóvenes ni de los países en general.

Sobre el tema particular de la transición a la vida adulta, la concepción tradicional considera que la adultez libera a los jóvenes de su condición juvenil al brindarles la posibilidad de emanciparse del hogar parental, ser autónomos e independientes financieramente, así como en algún momento tener la oportunidad de formar su propia familia (Pérez-Islas, 2009). Al respecto, la CEPAL (2008) establece que "la autonomía suele atribuirse casi como un valor natural de la juventud; sin embargo, no es tan fácil de construir como proyecto efectivo de vida, dadas las barreras al empleo y la vivienda [...]" (p. 11). De acuerdo con esto, conseguir trabajo remunerado no asegura poder independizarse completamente de los padres. Hay personas que han rebasado la edad juvenil convencional pero no "han alcanzado plenamente el estatus de adulto" (Revilla, 2001, p. 118). Lo anterior diversifica los trayectos rumbo a la vida adulta que vuelven relevante seguir indagando sobre este tema de la emancipación juvenil ligado estrechamente al trabajo.

El trabajo es un factor determinante de las cuatro transformaciones históricas de lo juvenil (Pérez-Islas, 2009). En la primera, el individuo es separado gradualmente del hogar familiar -el cual era también un espacio comunitario de aprendizaje y de producción económica-. En esta etapa del capitalismo preindustrial del siglo XV hasta la primera mitad del XVIII (Barnes, 1987), familia, escuela y trabajo emergen como los espacios institucionales encargados de diferentes misiones sociales. A la familia se le encomendaría dar cabida a los afectos y emociones; la escuela asumiría la responsabilidad de administrar conocimientos y saberes; y, por último, el trabajo sería el territorio asignado para realizar actividades productivas. A partir del tránsito del sistema feudal hacia el capitalismo comienzan a surgir las categorías de trabajador, asalariado, y también, la categoría de joven (Pérez-Islas, 2009).

La creciente estructuración espacial y temporal de las actividades de los individuos acarreada por la Revolución Industrial del siglo XVIII (Barnes, 1987) originó una segunda transformación histórica de lo juvenil. En la escuela se socializaba a los individuos y después comenzó a formárseles para el mundo del trabajo. Además de los conocimientos adquiridos en la escuela, los jóvenes, agrupados por edad, comenzaron a 
convivir con personas de su misma generación; esto dio "un nuevo impulso a la construcción de lo juvenil" (Pérez-Islas, 2009, p. 31). La formación para el trabajo se obtenía en la escuela y, posteriormente, se volvió un requisito para ingresar al mundo laboral; por lo tanto, la preparación para el trabajo delineó el rumbo hacia la vida adulta. La vida juvenil iniciaba en el hogar, hacía escala forzosa en la escuela y concluía cuando el individuo se convertía en trabajador.

El empleo, concebido como trabajo asalariado, marca el inicio de la tercera transformación (Pérez-Islas, 2009). El auge promovido por los modelos de fabricación en serie -que predominaron como esquemas de producción de mercancías gran parte del siglo $\mathrm{XX}-$, posicionó a las actividades industriales como los principales espacios productivos capaces de ofrecer trabajo a los jóvenes. En esta fase del capitalismo que inicia a fines del primer tercio del siglo para extenderse hasta la década de 1970 (Barnes, 1987), coinciden las cadenas de ensamble de las fábricas con la "normalización y estandarización de lo juvenil" (Pérez-Islas, 2009, p. 32), ya que, además de la emancipación familiar, encontrar un empleo representaba el pase directo para formar parte de la estructura social (el trabajo traía consigo ingreso económico, así como derechos y responsabilidades propias de los ciudadanos de mayor edad). En otras palabras, “la condición juvenil se vinculó entonces a la posibilidad o no de tener un empleo como frontera de inserción o exclusión de los beneficios de la sociedad y la ciudadanía" (Pérez-Islas, 2009, p. 32). En consecuencia, asegurar la empleabilidad se instauró como una de las preocupaciones más apremiantes de personas jóvenes y adultas.

La entrada de los jóvenes al mundo laboral inauguraba también su ingreso a la vida adulta. No obstante, cambios ocurridos en la esfera del empleo a nivel global (relación contractual, flexibilización de horarios, deslocalización y fragmentación de actividades productivas, etc.), han modificado la idea de que la inserción laboral representa el punto de clausura de la juventud. El trabajo solía ser el medio a través del cual los jóvenes lograban emanciparse del hogar parental y alcanzaban independencia económica, para a su vez, comenzar su propio ciclo social y productivo como sujetos autónomos (Pérez-Islas, 2009). Esta discordancia entre inserción laboral y condición de adulto es uno de los fenómenos medulares que influyen en las trayectorias de vida de los jóvenes contemporáneos. Dicha discordancia o desajuste corresponde a lo que Pérez-Islas (2009) nombra la "cuarta transformación" (p. 32) histórica de la condición juvenil, la cual se desprende de la serie de cambios socioeconómicos y culturales que marcaron el rumbo de la economía mundial desde el último cuarto del siglo 
XX hasta nuestros días (Escalante, 2015). Teniendo en cuenta lo anterior, Pérez-Islas (2009) formula la "hipótesis de que una cuarta transformación está en curso (llámese crisis de la sociedad salarial, precarización del empleo, tercerización o subcontratación, tecnologización, informatización, flexibilización y todos los pos-fordismo, taylorismo, neoliberalismo) y afecta directamente a la condición juvenil contemporánea" (p. 32).

Esa cuarta transformación de lo juvenil ha causado tres rupturas. La primera afecta capacidad de decisión y agencia de los jóvenes: al disminuir las oportunidades, también decrece la posibilidad de actuar y tomar decisiones. La segunda ruptura trastorna su proceso de emancipación. La precarización laboral y el desempleo han dificultado el desprendimiento familiar habitacional y financiero; tal situación ha despertado nuevos debates sobre la delimitación etaria de la juventud y el ingreso a la etapa adulta. La tercera ruptura se relaciona con procesos reflexivos y perspectivas a futuro de las generaciones jóvenes (Pérez-Islas, 2009).

Estas rupturas históricas también han influido las discusiones académicas actuales sobre lo juvenil; ello ha motivado repensar y cuestionar el "significado social" (Pérez-Islas, 2009) de la edad como frontera que separa al joven del adulto. En ese sentido, han surgido replanteamientos de los conceptos de juventud y adultez, concepciones como las de "juventud cuasi permanente" (Pérez-Islas, 2009), adultez semi autónoma o dependiente, son conceptos con los cuales se intentan explicar las transformaciones de lo juvenil derivadas de la crisis que experimenta el mundo del trabajo en el siglo XXI. Un nuevo contrato social está desplazando al esquema del empleo remunerado, lo cual, a su vez, transforma la transición a la vida adulta de las nuevas generaciones de jóvenes (Pérez-Islas, 2009). Efectos de ese nuevo contrato social se pueden encontrar en el diagnóstico realizado por la CEPAL y la Organización Iberoamericana de Juventud (OIJ) en 2007, en el cual pronosticaban los principales problemas que estarían enfrentando en el corto plazo los jóvenes iberoamericanos de entre 15 y 29 años. De acuerdo con el informe, los jóvenes ampliarían su acceso a la educación, pero las oportunidades laborales serían escasas. Esto conllevaría un efecto negativo en el valor de la educación, donde la inversión en formación académica no garantizaría obtener un empleo que retribuyera ingreso igual o superior a dicha inversión.

En otras latitudes se han encontrado controversias en la relación educación-inserción laboral. Por ejemplo, en España, desde antes de la crisis económica global detonada por el colapso financiero de 2008, "un elevado nivel educativo ya había dejado de tener el papel de protección frente a la inestabilidad" (Verd y López-Andreu, 2016, p. 13). El nivel de escolaridad ha perdido eficacia para asegurar un buen trabajo; ello "pondría 
de manifiesto la importancia de otros capitales, como el cultural o el relacional, en las trayectorias laborales" (Verd y López-Andreu, 2016, p. 13). En este sentido, la CEPAL (2008) afirma que "las posibilidades de acceder a los segmentos que prometen mejores condiciones de empleo y trayectorias laborales ascendentes dependen del capital humano, capital social y capital cultural de los jóvenes" (p. 171). A partir de estos argumentos se puede observar cómo el exceso de educación no ha representado un factor eficaz para encontrar o conservar empleos dignos. Además, el fenómeno descrito no es reciente y de cierta manera presiona a los jóvenes a desarrollar diversos capitales simbólicos; al mismo tiempo los apremia a movilizar conocimientos, habilidades y actitudes para evitar quedar rezagados o excluidos de las ofertas laborales.

Siguiendo con el tema de la emancipación, cabe resaltar que el diagnóstico de la CEPAL y la OIJ (2007) advierte sobre la tendencia a la "autodeterminación y protagonismo" (p. 21), por un lado; y, por otro, a la "precariedad y desmovilización" (p. 21); dos polos opuestos convertidos en factores causantes de las principales tensiones vislumbradas en el panorama juvenil actual. Esto quiere decir que los jóvenes mantendrían expectativas elevadas para independizarse y ser autónomos; sin embargo, en realidad tendrían pocas alternativas a su alcance para lograrlo. La escasez de oportunidades laborales, la exigencia creciente de formación y experiencia por parte de los empleadores, así como el deterioro de las condiciones laborales dificultarían la independencia económica y la emancipación familiar, ralentizando así la integración de los jóvenes hacia la condición social adulta. Lo anterior confirma y pone en el centro a los jóvenes como uno de los segmentos poblacionales más afectados por las características del modelo de trabajo predominante en el siglo XXI.

\section{Flexibilidad laboral y juventudes latinoamericanas}

Hasta aquí hemos visto que entre las consecuencias más preocupantes de los procesos de globalización económica se encuentra la creciente incertidumbre y precarización de los entornos laborales formales; "el plano en el cual se desarrollan estos procesos de transformación social está delimitado por la globalización y un nuevo modelo de acumulación en América Latina, caracterizado por la desregulación y la flexibilidad laboral" (Mancini en Mora y Oliveira, 2014, p. 147). En América Latina, los jóvenes conviven con situaciones adversas de diferente índole que caracterizan a las sociedades de la región, entre ellas: dificultades económicas y laborales, problemas familiares, violencia, adicciones, falta de oportunidades, 
discriminación, vulnerabilidad, injusticia, fragilidad existencial y tensión en las relaciones sociales. Se observa también cómo los desafíos y retos enfrentados por este grupo etario van en aumento, en relación inversa con las oportunidades laborales disponibles para ellos. Esta situación dificulta la emancipación juvenil y deteriora las condiciones de llegada a la etapa adulta, situación que, a la larga, perjudica el desarrollo económico de un país, la calidad de vida y las perspectivas de futuro de los integrantes de la sociedad.

Las disposiciones de austeridad adoptadas por gran cantidad de países latinoamericanos para hacer frente a la crisis económica de la primera década del siglo XXI impactaron principalmente en las condiciones de los empleos (Contreras, 2015; Escalante, 2015); ello ocasionó un entorno de precariedad laboral. Tres de las características más significativas de ese entorno son: descenso generalizado de los salarios, desaparición gradual de prestaciones y menoscabo de los derechos laborales (Gómez, 2012). Las medidas flexibilizadoras del trabajo involucraron primordialmente: alargamiento de jornadas, congelamiento o lento avance de los salarios, así como paulatina desaparición de derechos y recompensas laborales. En cuanto al incremento salarial, éste se ha visto mermado en los países con gobiernos de corte neoliberal. Dicho crecimiento salarial -poco significativo- ha sido acompañado de aumentos continuos en los precios que, aunque controlados, no han cesado; esto repercute de forma directa en el poder adquisitivo (Escalante, 2015). Con base en el panorama descrito, las estrategias flexibilizadoras del trabajo pueden ser entendidas como una forma legal para explotar a los individuos oriundos de países que decidieron desproteger a su mano de obra como remedio para atraer capitales extranjeros

El fenómeno flexibilizador no afecta exclusivamente a los jóvenes; expulsa también del sistema formal laboral a gran cantidad de adultos quienes, en su gran mayoría, encuentran en el autoempleo su única alternativa. Las personas que deciden trabajar en el sector económico informal -residuo o "producto del sistema capitalista de producción" (Gómez, 2012, p. 30)- conforman el "ejército de reserva de la economía capitalista" o "reservas de fuerza laboral" (Gómez, 2012, p. 30). Se le nombra así al conjunto de individuos con deseos o interés en reintegrarse en cualquier momento al sistema formal; aceptando iguales o peores condiciones laborales con tal de tener un ingreso seguro y evadir -aunque sea de manera indefinida- la incertidumbre. En el contexto actual, el sector juvenil constituye un segmento poblacional dispuesto a trabajar en las condiciones precarias ofertadas por el mercado de recursos humanos.

Para ahondar en este tema de la precarización laboral de los jóvenes 
en Latinoamérica, consultamos la investigación de Jiménez (2012), donde compara cómo las condiciones laborales en México y Argentina se han deteriorado. De acuerdo con el estudio, los efectos del trabajo en las biografías de los individuos se manifiestan en diferentes frentes; uno de ellos es la estructuración de las vidas cotidianas de los sujetos -en particular lo que se refiere a la construcción de lazos intersubjetivos ya sean de carácter familiar o social.

Las distintas formas adoptadas por el trabajo han originado nuevas significaciones de lo que actualmente los jóvenes ven como una jornada laboral (Jiménez, 2012). Por ejemplo, los jóvenes ahora se enfrentan a trabajos cuya duración es todo el día y toda la semana; estas opciones laborales son factibles debido a la conectividad constante lograda a través de nuevos dispositivos electrónicos que permiten o facilitan el acceso a internet. Las expectativas de que la gente sea multitarea, multiactividad y estar siempre disponible corresponden a las nuevas dinámicas laborales del siglo XXI.

Para finalizar, queremos resaltar que, en el caso de Latinoamérica han brotado grupos de jóvenes sin muchos problemas para adaptarse a las nuevas condiciones y exigencias laborales del nuevo siglo. A la par de que conservan ciertos valores tradicionales (deseos por independizarse del hogar parental, lograr la autonomía económica, formación de su propia familia, viajes y planes a futuro, etc.), han asimilado ideas y preocupaciones sociales que han ganado terreno en las últimas décadas, como la equidad de género y la vulnerabilidad laboral (Gómez, 2012). La mayor duración de las jornadas y el aumento de requisitos y actividades de los empleos formales han generado tensiones hacia dentro de los núcleos familiares, mismos que se han visto desplazados o descuidados por la necesidad de asegurar el ingreso económico. Esta situación, junto con el desempleo, ha motivado o forzado a muchos sujetos a generar su propia fuente de ingreso mediante el autoempleo; esquema de emprendimiento individual o colaborativo que ha ganado terreno como alternativa a la organización tradicional de tiempo y espacio de los trabajos.

\section{Consideraciones}

La precarización de las trayectorias juveniles es uno de los factores que ha provocado que las fronteras entre adultos y jóvenes se hayan tornado borrosas. De ahí que la emancipación de los jóvenes tarde en llegar o simplemente no llega; algunas veces por voluntad propia, otras porque los trabajos y los ingresos que se obtienen no hacen viable una vida digna fuera del hogar parental. Hay también jóvenes que viven en domicilios distintos 
al de sus padres, pero no cortan del todo los vínculos de dependencia y que enfrentan grandes retos para lograr la emancipación de su núcleo familiar.

La revisión realizada nos coloca ante un aspecto de carácter teórico de gran relevancia, nos referimos a que la noción de trabajo desarrollada durante el siglo XIX y la primera mitad del siglo XX se ha transformado de manera dramática para las nuevas generaciones. Si el siglo XIX fue el siglo de los sindicatos y de los partidos de izquierda en donde se dieron grandes batallas para defender los derechos de los trabajadores, en la actualidad pareciera ser que los jóvenes están solos en este proceso; es decir, ya no se cuenta con el respaldo de sindicatos ni de partidos políticos (quienes han sacado de sus agendas estos temas); por lo tanto, los jóvenes tienen que velar ellos mismos por crear sus condiciones y sus propios espacios de trabajo. Para muchos jóvenes no hay inclusión y tampoco hay igualdad de oportunidades. Se enfrentan a la ausencia de la seguridad social (Prieto y Vega, 2018).

Las condiciones que imperan en el mercado laboral hacen que sea muy poco realista la posibilidad de "acomodar" a todos los jóvenes de manera digna, decente y eficaz en espacios laborales. Sobre esto último, es importante destacar que los jóvenes que son trabajadores por cuenta propia se han visto en la necesidad de improvisar y hacer de cualquier espacio un espacio para trabajar. En las ciudades cada vez con mayor frecuencia somos testigos del crecimiento de los llamados coworkings; espacios que se pueden rentar por hora, día, semanas, meses y representan una alternativa espacial para los nuevos profesionistas independientes. Son centros que permiten interacción y conectividad, lo cual facilita la construcción de plataformas colaborativas; obteniendo con ello la posibilidad de generar espacios para crear comunidad. Lo anterior, posibilita que se puedan potenciar ciertas competencias o capacidades de los jóvenes, tales como: interacción, creatividad y colaboración.

Sin embargo, esta nueva pauta o estos nuevos escenarios desencadenados por la transformación laboral no han dejado de lado condiciones de precariedad, aunque los jóvenes cuenten con nuevas habilidades-competencias y recursos tecnológicos a la mano. Más bien lo que vemos son experiencias laborales sin prestaciones, con altas dosis de estrés, en las que los jóvenes aprenden a sobrevivir a partir de desarrollar múltiples proyectos laborales a la vez. Ciertamente los dispositivos tecnológicos de última generación y el desarrollo de las redes sociales permiten que muchos trabajos puedan realizarse desde cualquier lugar, pero en cambio, debemos de cuestionarnos acerca de la calidad de vida y de los trabajos a los que están accediendo las nuevas generaciones. 
Estamos siendo testigos del surgimiento de nuevas formas de empleo y muchas de ellas muy relacionadas a las nuevas tecnologías de la información. Además de que se hace evidente de que el mercado de trabajo requiere de personas que cuenten con las siguientes competenciashabilidades: capacidad de rápida adaptación, flexible, buena comunicación, contar con capacidad de diálogo, capacidad de negociación, pensamiento asertivo y facilidad para plantear y resolver problemas. Además, estas competencias deben ser polivalentes y siempre actualizadas, como si de conocimientos técnicos se trataran. En consecuencia, los jóvenes deben hacerse empleables a sí mismos. Además, al deseo de trabajar, los jóvenes tendrán que sumarle motivación para buscar empleo, capacidad de saber buscarlo, disposición para adquirir la información necesaria, capacidad de esfuerzo para mejorar las competencias como trabajador y, además, tener una actitud positiva, ánimo, autodisciplina, afán de superación, confianza en uno mismo, entre otras cuestiones.

Sin duda el mayor reto para los gobiernos de América Latina es abatir el enorme déficit que en condiciones de justicia laboral enfrentan amplios sectores de la población y en particular de los jóvenes. Se requieren acciones para lograr empleos que otorguen dignidad humana y que se aseguren los derechos de los trabajadores. El reto es lograr mayores empleos con condiciones decentes y que logren incorporar a la mayor parte de aquellos jóvenes que se mueven fuera de las redes de la protección social. Somos testigos de que los tiempos actuales han generado formas atípicas de empleo. Las relaciones contractuales, los tiempos, el lugar de trabajo, las remuneraciones e incluso los horarios ya no están claramente definidas y todo lo anterior es mucho más evidente para los jóvenes.

Finalmente, es un hecho que los empleos de larga duración y con un sistema de protección de los derechos laborales son cada vez menos y sólo pequeñas minorías tienden y tendrán acceso a ellos. Es decir, aquellas experiencias de un trabajo para toda la vida son experiencias que cada vez es menos probable que se presenten para los jóvenes y, cabría preguntarse si es lo que ellos en realidad buscan. 


\section{Referencias bibliográficas}

Barnes, H. (1987). Historia de la economía del mundo occidental. México, D.F.: UTEHA.

Bazán, L. (1996). Cuando una puesta se cierra, abrimos cientos. Estrategias de las familias petroleras frente al cierre de la refinería 18 de marzo. México: UNAM.

Castillo D., Baca, N. y Todaro, R. (2016). Trabajo global y desigualdades en el mercado laboral. México: Universidad Autónoma del Estado de México, CLACSO.

Comisión Económica para América Latina y el Caribe (CEPAL). (2008). Juventud y cohesión social en Iberoamérica. Un modelo para armar. Santiago: Chile. Recuperado de http:// repositorio.cepal.org/handle/11362/3639.

Comisión Económica paraAmérica Latina yelCaribe(CEPAL) y Organización Iberoamericana de Juventud (OIJ). (2007). La juventud en Iberoamérica: Tendencias y urgencias. Santiago, Chile. Recuperado de http://repositorio.cepal.org/handle/11362/2785.

Contreras, M. (2015). Crítica a la razón neoliberal: del neoliberalismo al posliberalismo. México, D.F.: Akal.

Escalante, F. (2015). Historia mínima del neoliberalismo. México, D.F.: El Colegio de México.

Estrada, M. (1996). Después del despido. Desocupación y familia obrera. México: CIESAS.

Feixa, C. (1999). Antropología de las edades. Publicación virtual /Biblioteca virtual de Ciencias Sociales. Recuperado de http://www.cholonautas.edu.pe/modulo/upload/C\%20Feixa. pdf.

Feixa, C. (2006). Teorías sobre la juventud en la era contemporánea. Revista Latinoamericana de Ciencias Sociales, Niñez y Juventud, 4(2), 2-18.

García, B. y Oliveira, O. (1994). Trabajo femenino y vida familiar en México. México: El Colegio de México.

García, B. (1981). Un pueblo fabril en el porfiriano, Santa Rosa, Veracruz. México: Siglo XXI.

Gil Calvo, E. (2005). El envejecimiento de la juventud. Revista de Estudios de Juventud (71), 11-19.

Gómez, M. (2012). Los jóvenes ante la precariedad laboral. En M.L. Jiménez y R. Boso (coord.), Juventud precarizada. De la formación al trabajo, una transición riesgosa (pp. 25-46). Cuernavaca: UNAM, Centro Regional de Investigaciones Multidisciplinarias.

González, M. (1986). Los recursos de la pobreza. Familias de bajos ingresos en Guadalajara. México: El Colegio de Jalisco / CIESAS / SPP.

Gutmann, M. (1993). Los hombres cambiantes, los machos impenitentes y las relaciones de género en el México de los noventa. Estudios Sociológicos, XI (33), 725 - 740.

Jiménez, M. (2012). Introducción. En M.L. Jiménez y R. Boso (coord.), Juventud precarizada. De la formación al trabajo, una transición riesgosa (pp. 9-24). Cuernavaca, México: UNAM, Centro Regional de Investigaciones Multidisciplinarias.

Lewis, Ó. (2012). Los hijos de Sánchez. Una muerte en la familia Sánchez. México: Fondo de Cultura Económica.

Macri, M. (1999). Estudiar y trabajar: perspectivas y estrategias de los adolescentes. Argentina: Ediciones La Crujia.

Mancini, F. (2014). El impacto de la incertidumbre laboral sobre el curso de vida durante la transición a la adultez. En M. Mora y O. de Oliveira (coord.), Desafíos y paradojas. Los jóvenes frente a las desigualdades sociales (pp. 147-187). México, D.F.: El Colegio de México.

Mancini, F. (2017). Asir incertidumbres. Riesgo y subjetividad en el mundo del trabajo. México: ISS/UNAM/El Colegio de México.

Nieto, R. (1988). Espacio laboral y trabajo. En Bazán et al. (eds.), La situación de los obreros del calzado en León (pp. 65-116). México: CIESAS. 
Novelo, V. y Urteaga, A. (1980). La industria en los magueyales. México: CIS-INAH, Nueva Imagen.

Oliveira, O. y Salles, V. (1989). Introducción. En O. De Oliveira., M. Lehalleur y V. Salles (comp.), Grupos domésticos y reproducción cotidiana (pp. 11-36). México: El Colegio de México/Miguel Ángel Porrúa.

Organización Internacional del Trabajo (OIT). (2015). Tendencias mundiales del empleo juvenil 2015. Promover la inversión en empleos decentes para los jóvenes. Suiza.

Pérez-Islas, J.A. (2009). Las cuatro grandes transformaciones históricas de la condición juvenil. Suplemento Diario de Campo, (56), 29-35.

Prieto, M. y Vega L. (2018). El futuro del trabajo: ¿amanecer u ocaso?. Trabajo (15), 11-36.

Revilla, J. (2001). La construcción discursiva de la juventud: lo general y lo particular. Papers, $63 / 64,102-122$.

Rivera, J. (2004). Reestructuración y crisis económica: un estudio sobre las nuevas formas de vida y trabajo en un grupo de familias de los sectores medios en la ciudad de México. México: Universidad Autónoma Metropolitana/Unidad Iztapalapa.

Safa, H. (1999). Prólogo. En M. González (coord.), Divergencias del modelo tradicional: Hogares de jefatura femenina en América Latina (pp. 9-17). México: CIESAS/ PYV.

Saraví, G. (2009). Transiciones vulnerables. Juventud, desigualdad y exclusión en México. México: CIESAS.

Thrasher, F. (1963) [1927] The Gang. A Study of 1313 Gangs in Chicago, Chicago, University of Chicago Press.

Trejo, J., Arzate, J. y Palermo, A. (2010). Desigualdades sociales y. Ciudadanía desde las culturas juveniles en América. Latina. México: Miguel Ángel Porrúa/Universidad Autónoma del Estado de México.

Urteaga, M. (2009). Juventud y antropología: una exploración de los clásicos. Suplemento Diario de Campo (56), 13-27.

Verd, J. y López-Andreu, M. (2016). Crisis del empleo y polarización de las trayectorias laborales. El caso de los adultos jóvenes en Cataluña. Papers, 101(1), 5-30.

Weller, J. (2007). La inserción laboral de los jóvenes: características, tensiones y desafíos. Revista de la CEPAL, 92, 61-82.

Whyte, W. (1971). La sociedad de las esquinas. México, D.F.: Diana. 